Gazi University
Journal of Science
$\mathrm{http} / /$ dergipark.gov.tr/gujs

\title{
Local $T_{3}$ Constant Filter Convergence Spaces
}

\author{
Tesnim Meryem BARAN ${ }^{1}$ (D) , Ayhan ERCIYES ${ }^{2, *}$ \\ ${ }^{1}$ Ministry of National Education, Pazaroren Anatolian High School, 38730, Kayseri, Turkey \\ ${ }^{2}$ Aksaray University, Faculty of Arts and Sciences, Department of Mathematics, 68100, Aksaray, Turkey
}

\section{Highlights}

- We characterized each of local $\bar{T}_{3}$ (resp. $T_{3}{ }^{\prime}, S \bar{T}_{3}, S T_{3}{ }^{\prime}$ ) constant filter convergence spaces.

- We investigated the relationships among these various forms.

- We showed that the categories $\bar{T}_{3} \mathrm{ConFCO}$ and $\mathrm{ST}_{3} \mathrm{ConFCO}$ were isomorphic categories.

- We showed that the categories $T_{3}{ }^{\prime} \mathrm{ConFCO}$ and $\mathrm{ST}_{3}{ }^{\prime} \mathrm{ConFCO}$ were isomorphic categories.

\section{Article Info}

Received: 05/08/2019 Accepted: $24 / 12 / 2019$

\section{Keywords}

Topological category Convergence spaces PreHausdorff spaces $T_{3}$ spaces

\begin{abstract}
In this paper, we characterize each of local $T_{3}\left(\right.$ resp. $\left.T_{3}{ }^{\prime}, S \bar{T}_{3}, S T_{3}{ }^{\prime}\right)$ constant filter convergence spaces and investigate the relationships among these various forms. We show that the full subcategories $\bar{T}_{3}$ ConFCO and $S \bar{T}_{3}$ ConFCO (resp. $T_{3}{ }^{\prime} \mathrm{ConFCO}$ and $\mathrm{ST}_{3}{ }^{\prime} \mathrm{ConFCO}$ ) of ConFCO are isomorphic categories. Moreover, we show that if a constant filter convergence space $(B, K)$ is $\bar{T}_{3}$ (resp. $T_{3}^{\prime}, S \bar{T}_{3}$ or $S T_{3}{ }^{\prime}$ ) at $p$ and $M \subset B$ with $p \in M$, then $M$ is $\bar{T}_{3}$ (resp. $T_{3}^{\prime}$ ) at $p$.
\end{abstract}

\section{INTRODUCTION}

Filters are first defined in the papers of Cartan [1,2] and play an important role in defining convergence in a manner similar to the role of sequences in a metric space. In 1978, Schwarz [3] introduced the category of constant filter convergence spaces which is isomorphic to the category of Grill spaces.

In 1991, Baran [4] gave a generalization of local $T_{0}$ and $T_{1}$ axioms of topology to topological categories. Local $T_{2}$ objects are defined in terms of local $T_{0}$ objects [4] and local $T_{1}$ are used to define the notion of closedness [5] in arbitrary topological categories. Furthermore, local $T_{1}$ is used to define the local $T_{3}$ and $T_{4}$ separation properties in arbitrary topological categories [4].

\section{PRELIMINARIES}

Let $B$ be a non-empty set and $F(B)$ be the set of filters on $B$. A filter $\alpha \in F(B)$ is called proper (improper) iff $\emptyset \notin \alpha$ (resp. $\emptyset \in \alpha)$.

$(B, K)$ is called a constant filter convergence space if the map $K: B \rightarrow P(F(B))$ satisfies:

(1) $[x] \in K, \forall x \in B$, where for $U \subset B$ and $[U]=\{V \subset B: U \subset V\}$,

(2) if $\alpha \in K$ and $\alpha \subset \beta$, then $\beta \in K$.

Let $(X, K)$ and $(Y, L)$ be constant filter convergence spaces and $f: X \rightarrow Y$ be a function. Then $f$ is said to be continuous if for any $\alpha \in K$ implies $f(\alpha) \in L$, where 
$f(\alpha)=\{U \subset X: \exists A \in \alpha$ such that $f(A) \subset U\})$.

Let $\boldsymbol{C o n F C O}$ be the category of constant filter convergence spaces and continuous maps [3]. Note that the category $\operatorname{ConFCO}$ is a normalized topological category [6].

Definition 2.1. A source $\left\{f_{i}:(B, K) \rightarrow\left(B_{i}, K_{i}\right), i \in I\right\}$ in ConFCO is an initial lift if and only if $\alpha \in K$ precisely when $f_{i}(\alpha) \in K_{i}$ for all $i \in I$ [7].

Definition 2.2. An epi $\operatorname{sink}\left\{f_{i}:\left(B_{i}, K_{i}\right) \rightarrow(B, K), i \in I\right\}$ is final if and only if $\alpha \in K$ implies there exists $\beta_{i} \in K_{i}$ such that $f_{i}\left(\beta_{i}\right) \subset \alpha[7]$.

Definition 2.3. Let $(B, K) \in \operatorname{ConFCO} . K=\{[a], P(B)=[\varnothing]: a \in B\}$ is the discrete structure on $B$.

\section{LOCAL $T_{3}$ CONSTANT FILTER CONVERGENCE SPACES}

In this section, we give the characterization of local $T_{3}$ constant filter convergence spaces and find out relationships amoung them.

Let $B$ be set with $p \in B$ and $B \bigvee_{p} B$ be the wedge at $p$ [4]. Define

$S_{p}: B \vee_{p} B \rightarrow B^{2}$ by $S_{p}\left(x_{i}\right)=\left\{\begin{array}{ll}(x, x), & i=1 \\ (p, x), & i=2\end{array}\right.$,

$A_{p}: B \vee_{p} B \rightarrow B^{2}$ by $A_{p}\left(x_{i}\right)=\left\{\begin{array}{ll}(x, p), & i=1 \\ (p, x), & i=2\end{array}\right.$ and

$\nabla_{P}: B \vee_{p} B \rightarrow B$ by $\nabla p\left(x_{\mathrm{i}}\right)=x$ for $i=1,2$,

where $x_{1}\left(\right.$ resp. $\left.x_{2}\right)$ is in the first (resp. second) component of $B \bigvee_{p} B[4,5]$.

Definition 3.1. ([4,5]) Let Set be the category of sets and functions, $U: \mathcal{E} \rightarrow$ Set be a topological functor, and $X$ be an object of $\mathcal{E}$ with $p \in U(X)=B$.

(1) If the initial lift of the $U$-source $\left\{S_{p}: B \bigvee_{p} B \rightarrow U\left(X^{2}\right)=B^{2}\right.$ and $\left.\nabla_{P}: B \bigvee_{p} B \rightarrow U D(B)=B\right\}$ is discrete, then $X$ is called $T_{1}$ at $p$, where $D$ is discrete functor,

(2) If the initial lift of the $U$-source $S_{p}: B \bigvee_{p} B \rightarrow U\left(X^{2}\right)=B^{2}$ and $A_{p}: B \bigvee_{p} B \rightarrow U\left(X^{2}\right)=B^{2}$ is agree, then $X$ is called $\operatorname{Pre} \bar{T}_{2}$ at $p$,

(3) If the initial lift of the $U$-source $S_{p}: B \bigvee_{p} B \rightarrow U\left(X^{2}\right)=B^{2}$ and the final lift of the $U$-sink $i_{1}, i_{2}$ : $U(X)=B \rightarrow B \bigvee_{p} B$ is agree, then $X$ is called $\operatorname{PreT}_{2}{ }^{\prime}$ at $p$, where $i_{1}, i_{2}$ are the canonical injections.

Remark 3.2. Let $(B, \tau)$ is a topological space and $p \in B$. $\operatorname{Pre}_{2}{ }^{\prime}$ and $\operatorname{Pre} \bar{T}_{2}$ at $p$ are equivalent and reduces to every $x \in X$ with $x \neq p$, the topological space $(\{x, p\}, \delta)$ is not indiscrete, then the points $x$ and $p$ have disjoint neighborhoods [8].

Definition 3.3. ([4]) Let $U: \mathcal{E} \rightarrow$ Set be a topological functor, $X$ is an object of $\mathcal{E}$ with $p \in U(X)$ and $X / F$ be the final lift of the epi $U$-sink

$$
q: U(X)=B \rightarrow B / F=(B \backslash F) \cup\{*\},
$$

where $q$ is the identity on $B \backslash F$ and identifying $F$ with a point * [4]. 
(1) If $X$ is $T_{1}$ at $p$ and $X / F$ is $\operatorname{Pr} e \bar{T}_{2}$ at $p$ for every non-empty closed $F$ in $U(X)$ missing $p$, then $X$ is called $\bar{T}_{3}$ at $p$,

(2) If $X$ is $T_{1}$ at $p$ and $X / F$ is $\operatorname{PreT}_{2}{ }^{\prime}$ at $p$ for $\emptyset \neq F \subset U(X)$ closed with $p \notin F$, then $X$ is called $T_{3}{ }^{\prime}$ at $p$,

(3) If $X$ is $T_{1}$ at $p$ and $X / F$ is $\operatorname{Pr} \bar{T}_{2}$ at $p$ for $\emptyset \neq F \subset U(X)$ closed with $p \notin F$, then $X$ is called $S \bar{T}_{3}$ at $p$,

(4) If $X$ is $T_{1}$ at $p$ and $X / F$ is $\operatorname{PreT}_{2}{ }^{\prime}$ at $p$ for $\emptyset \neq F \subset U(X)$ closed with $p \notin F$, then $X$ is called $S T_{3}{ }^{\prime}$ at $p$.

Note that if $(B, \tau)$ is a topological space and $p \in B$, then by Theorem 2.1 of [8], all of $T_{3}{ }^{\prime}$ at $p, \bar{T}_{3}$ at $p, S T_{3}{ }^{\prime}$ at $p$, and $S \bar{T}_{3}$ at $p$ are same.

Remark 3.4. Let $\alpha, \beta \in F(A)$ and $f: A \rightarrow B$ be a function. Then

(1) $f(\alpha \cap \beta)=f(\alpha) \cap f(\beta)$,

(2) $f(\alpha) \cup f(\beta) \subset f(\alpha \cup \beta)$,

(3) $f^{-1} f \alpha \subset \alpha$.

Lemma 3.5. ([9,10]) Let $B$ be a set, $\varnothing \neq F \subset B, \alpha, \beta, \sigma \in F(B)$, and $q: B \rightarrow B / F$ be identification map defined above.

(1) For $a \notin F, q \alpha \subset[a]$ iff $\alpha \subset[a]$,

(2) $q \alpha \subset[*]$ iff $\alpha \cup[F]$ is proper,

(3) $\alpha \cup[F]$ is not proper, then $q \sigma \subset q \alpha$ iff $\sigma \subset \alpha$,

(4) $\alpha \cup[F]$ is proper, then $q \sigma \subset q \alpha$ iff $\sigma \cup[F]$ is proper and $\sigma \cap[F] \subset \alpha$,

(5) $q \alpha \cup q \beta$ is proper iff $\alpha \cup \beta$ is proper or $\alpha \cup[F]$ and $\sigma \cup[F]$ are proper.

Theorem 3.6. Let $(B, K)$ be a constant filter convergence space with $p \in B$.

(1) $(B, K)$ is $T_{1}$ at $p$ iff $[x] \cap[p] \notin K, \forall x \in X$ with $x \neq p$,

(2) $(B, K)$ is $p r e \bar{T}_{2}$ at $p$ iff the conditions (i) and (ii) are satisfied, where

(i) If $\alpha, \beta \in K_{p}$, then $\alpha \cap \beta \in K_{p}$, where $K_{p}=\{\alpha: \alpha \subset[p]$ and $\alpha \in K\}$,

(ii) For any $\alpha \in K_{p}$ and $\beta \in K$ if $\alpha \cup \beta$ is proper, then $\beta \cap[p] \in K$,

(3) $(B, K)$ is $\operatorname{preT}_{2}{ }^{\prime}$ at $p$ if and only if $K_{p}=\{[p]\}$.

Proof. (1) (resp. (2)) is proved in [5] (resp. [11] ).

(3) Suppose $(B, K)$ is $\operatorname{preT}_{2}{ }^{\prime}$ at $p$ and $\alpha \in K$ with $\alpha \subset[p]$. In Theorem 3.15 of [10], let $\alpha_{1}=\alpha=\alpha_{2}$. Note that $\alpha_{1} \cup \alpha_{3}=\alpha$ is proper,

$$
\alpha_{1}=\alpha \supset \alpha_{3} \cap[p]=\alpha
$$


Hence by Theorem 3.15 of [9], we have a proper filter $\sigma$ on $B \bigvee_{p} B$ so that $\pi_{1} S_{p} \sigma=\alpha=\pi_{2} S_{p} \sigma$. Since $(B, K)$ is $\operatorname{preT}_{2}{ }^{\prime}$ at $p$, by Definition 3.1, $\sigma \supset i_{1} \sigma_{1}$ or $\sigma \supset i_{2} \sigma_{1}$ for some $\sigma_{1} \in K$.

If $\sigma \supset i_{1} \sigma_{1}$, then $\pi_{2} S_{p} \sigma=\alpha \supset \pi_{2} S_{p} i_{1} \sigma_{1}=[p]$ and consequently $\alpha=[p]$.

If $\sigma \supset i_{2} \sigma_{1}$, then $\pi_{1} S_{p} \sigma=\alpha \supset \pi_{1} S_{p} i_{1} \sigma_{1}=[p]$ and consequently $\alpha=[p]$. Hence, $K_{p}=\{[p]\}$.

Conversely, suppose $K_{p}=\{[p]\}$ and $\sigma$ is a filter on $B \bigvee_{p} B$, and $K_{S_{p}}$ be the constant filter structure on $B \bigvee_{p} B$ induced by $S_{p}$ and $K_{W}$ be structure on $B \vee_{p} B$ induced by the maps $i_{1}, i_{2}:(B, K) \rightarrow B \vee_{p} B$. We show that $K_{S_{p}}=K_{W}$.

Suppose $\sigma \in K_{S_{p}}$. By Definition 2.1, $\pi_{1} S_{p} \sigma \in K$ and $\pi_{2} S_{p} \sigma \in K$. In Theorem 3.15 of [9], let $\alpha_{1}=\pi_{1} S_{p} \sigma$ and $\alpha_{3}=\pi_{2} S_{p} \sigma$.

In case of (1) of Theorem 3.15 of [9], we have

$$
\pi_{1} S_{p} \sigma=[p] \text { and }\left(\pi_{1} S_{p} \sigma\right) \cup\left(\pi_{2} S_{p} \sigma\right)
$$

is improper. It follows easily that $\sigma \supset i_{2} \pi_{2} S_{p} \sigma$. Indeed, if $U \in i_{2} \pi_{2} S_{p} \sigma$, then $U \supset i_{2} \pi_{2} S_{p}(W)$ for some $W=U_{1} \vee_{p} U_{2} \in \sigma$. Since $\pi_{1} S_{p} \sigma=[p]$ and $\pi_{2} S_{p} \sigma \not \subset[p]$, we may assume $U_{1}=\emptyset$. Hence,

$$
W=U_{2}=i_{2} \pi_{2} S_{p}(W) \subset U
$$

and consequently, $U \in \sigma$ and $\sigma \supset i_{2} \pi_{2} S_{p} \sigma$.

In case of (2) of Theorem 3.15 of [9], $\pi_{1} S_{p} \sigma \not \subset[p]$ and $\pi_{1} S_{p} \sigma=\pi_{2} S_{p} \sigma$. By using similar argument above it is easy that $\sigma \supset i_{1} \pi_{1} S_{p} \sigma$.

In case of (3) of Theorem 3.15 of [9], we have

is proper and

$$
[p] \supset \pi_{1} S_{p} \sigma,\left(\pi_{1} S_{p} \sigma\right) \cup\left(\pi_{2} S_{p} \sigma\right)
$$

$$
\pi_{1} S_{p} \sigma \supset\left(\pi_{2} S_{p} \sigma\right) \cap[p]
$$

Note that $\pi_{1} S_{p} \sigma \in K,[p] \supset \pi_{1} S_{p} \sigma$ and by assumption, $\pi_{1} S_{p} \sigma=[p]$ and consequently, $\pi_{2} S_{p} \sigma=[p]$ since

is proper iff

$$
\left(\pi_{1} S_{p} \sigma\right) \cup\left(\pi_{2} S_{p} \sigma\right)=[p] \cup\left(\pi_{2} S_{p} \sigma\right)
$$

and

$$
[p] \supset \pi_{2} S_{p} \sigma
$$

$$
\pi_{2} S_{p} \sigma \in K
$$

Hence, $\sigma=\left[p_{1}\right]=i_{1}[p]$, where $p_{1} \in B \bigvee_{p} B$. Consequently, $\sigma \in K_{W}$ which shows that $K_{S_{p}} \subset K_{W}$. Suppose $\sigma \in K_{W}$. By Definition 2.2, there exists $\sigma_{1} \in K$ such that $\sigma \supset i_{1} \sigma_{1}$ or $\sigma \supset i_{2} \sigma_{1}$.

If $\sigma \supset i_{1} \sigma_{1}$, then

and

$$
\pi_{1} S_{p} \sigma \supset \pi_{1} i_{1} \sigma_{1}=\sigma_{1}
$$

$$
\pi_{2} S_{p} \sigma \supset \pi_{2} S_{p} i_{2} \sigma_{1}=\sigma_{1}
$$

and consequently $\pi_{i} S_{p} \sigma \in K, i=1,2$. By Definition 2.1, $\sigma \in K_{S_{p}}$. 
If $\sigma \supset i_{2} \sigma_{1}$, then

and

$$
\pi_{1} S_{p} \sigma \supset \pi_{1} S_{p} i_{2} \sigma_{1}=[p]
$$

$$
\pi_{2} S_{p} \sigma \supset \pi_{2} S_{p} i_{2} \sigma_{1}=\sigma_{1}
$$

and consequently $\pi_{i} S_{p} \sigma \in K, i=1,2$, i.e., $\sigma \in K_{S_{p}}$. Hence, $K_{W} \subset K_{S_{p}}$ and consequently $K_{W}=K_{S_{p}}$. By Definition 3.1, $(B, K)$ is $\operatorname{preT}_{2}{ }^{\prime}$ at $p$.

Lemma 3.7 Let $(B, K)$ be a constant filter convergence space and $\emptyset \neq F \subset B$. The following are equivalent:

(1) F is strongly closed,

(2) $F$ is closed,

(3) $\alpha \not \subset[a]$ or $\alpha \cup[F]$ is improper for any $a \in B$ with $a \notin F$ and $\forall \alpha \in K$.

Proof. It is proved in [5].

Theorem 3.8 Let $(B, K)$ be a constant filter convergence space with $p \in B$. The following are equivalent:

(1) $(B, K)$ is $S \bar{T}_{3}$ at $p$,

(2) $(B, K)$ is $\bar{T}_{3}$ at $p$,

(3) Conditions (i)-(iii) are satisfied, where

(i) For any $x \in B$ with $x \neq p,[x] \cap[p] \notin K$,

(ii) If $\alpha, \beta \in K_{p}$, then $\alpha \cap \beta \in K_{p}$, where $K_{p}=\{\alpha: \alpha \subset[p]$ and $\alpha \in K\}$,

(iii) For any $\alpha \in K_{p}, \beta \in K$ and $\emptyset \neq F \subset B$ closed with $p \notin F$, if $\alpha \cup \beta$ is proper or $\beta \cup[F]$ and $\alpha \cup[F]$ are proper, then $\beta \cap[p] \in K$.

Proof. By Lemma 3.7 and by Definition 3.3, a constant convergence space $(B, K)$ is $\bar{T}_{3}$ at $p$ iff $(B, K)$ is $S \bar{T}_{3}$ at $p$. Hence, $(1) \Leftrightarrow(2)$.

We need to show that $(2) \Leftrightarrow(3)$. Suppose $(B, K)$ is $\bar{T}_{3}$ at $p$. By Definition 3.3, in particular, $(B, K)$ is $T_{1}$ at $p$ and by Theorem 3.7(1), $[x] \cap[p] \notin K, \forall x \in B$ with $x \neq p$.

Suppose $\alpha, \beta \in K_{p}$. Then $q \alpha, q \beta \in K^{\prime}$ and $q \alpha \subset[p], q \beta \subset[p]$, where $K^{\prime}$ is the final constant filter structure on $B / F$. Since $(B, K)$ is $\bar{T}_{3}$ at $p$, by Definition $3.3,\left(B / F, K^{\prime}\right)$ is $\operatorname{pre}_{2}$ at $p$ for $\emptyset \neq F \subset B$ closed with $p \notin F$ and by Theorem 3.6(2),

$$
q(\alpha \cap \beta)=q(\alpha) \cap q(\beta) \in K_{p}{ }^{\prime} .
$$

By Definition 2.2, there exists $\delta \in K$ such that $q(\delta) \subset q(\alpha \cap \beta)$. Since $\alpha \cap \beta \subset[p]$ and $F$ is closed,. by Lemma 3.7,

$$
(\alpha \cap \beta) \cup[F]
$$

is improper and Lemma 3.5(3), $\delta \subset \alpha \cap \beta$ which shows that $\alpha \cap \beta \in K$ and consequently,

$$
\alpha \cap \beta \in K_{P} .
$$


Suppose that for any $\alpha \in K_{p}$ and $\beta \in K, \alpha \cup \beta$ is proper or $\beta \cup[F]$ and $\alpha \cup[F]$ are proper for $\emptyset \neq F \subset B$ closed with $p \notin F$. Note that $q \alpha, q \beta \in K^{\prime}$ and by Lemma $3.5(5), q \alpha \cup q \beta$ is proper and $q \alpha \subset[q(p)]=[p]$. Since $\left(B / F, K^{\prime}\right)$ is $\operatorname{pre}_{2} \bar{T}_{2}$ at $p$, by Theorem 3.6(2), $q(\alpha) \cap[p] \in K^{\prime}$. By Definition 2.2, there exists $\delta \in K$ such that

$$
q(\delta) \subset q(\beta) \cap[p]=q(\beta \cap[p]) .
$$

Since $\beta \cap[p] \subset[p]$ and $F$ is closed by Lemma 3.7, $(\beta \cap[p]) \cup[F]$ is improper and by Lemma 3.5(3), $\delta \subset \beta \cap[p]$ and consequently, $\beta \cap[p] \in K$. As a result, (iii) is proved.

Conversely, suppose that the conditions (i)-(iii) hold. By the condition (i) and Theorem 3.6(1), $(B, K)$ is $T_{1}$ at $p$. By Definition 3.3, we need to show that $\left(B / F, K^{\prime}\right)$ is $p r e \bar{T}_{2}$ at $p$ for $\emptyset \neq F \subset B$ closed with $p \notin F$, where $K^{\prime}$ is a structure on $B / F$. Suppose that $\alpha, \beta \in K^{\prime}$ with $\alpha \subset[p]$ and $\beta \subset[p]$. By Definition 2.2, $\alpha_{1}, \beta_{1} \in K$ such that

and

$$
q \alpha_{1} \subset \alpha \subset[p]=q[p]
$$

$$
q \beta_{1} \subset \beta \subset[p]=q[p] .
$$

Since $p \notin F, q \alpha_{1} \subset[p]$ and $q \beta_{1} \subset[p]$, by Lemma 3.5(1), we get $\alpha_{1} \subset[p]$ and $\beta_{1} \subset[p]$. By the condition (ii), $\alpha_{1} \cap \beta_{1} \in K_{p}$ and consequently, $\alpha \cap \beta \in K_{p}{ }^{\prime}$.

Now suppose that $\alpha \in K_{p}{ }^{\prime}$ and $\beta \in K^{\prime}$ with $\alpha \cup \beta$ is proper. By Definition 2.2, there exists $\alpha_{1}, \alpha_{2} \in K$ such that $q \alpha_{1} \subset \alpha, q \alpha_{2} \subset \beta$ and $q \alpha_{1} \subset[p]=[q(p)]$.

Since $\alpha \cup \beta$ is proper, then $q \alpha_{1} \cup q \alpha_{2}$ is proper and by Lemma 3.5(5), we have either $\alpha_{1} \cup \alpha_{2}$ is proper or $\alpha_{1} \cup[F]$ and $\alpha_{2} \cup[F]$ are proper. Note that $\alpha_{1} \subset[p]$ and $\alpha_{2} \in K$. If $\alpha_{1} \cup \alpha_{2}$ is proper, the by the condition (iii), we have $\alpha_{2} \cap[p] \in K$. So, $q\left(\alpha_{2} \cap[p]\right) \in K^{\prime}$, and $\beta \cap[p] \in K^{\prime}$.

Suppose $\alpha_{1} \cup[F]$ and $\alpha_{2} \cup[F]$ are proper. Since $F$ is closed, by Lemma 3.7, $\alpha_{1} \subset[p]$ and $\alpha_{2} \subset[p]$. By the condition (ii), $\quad \alpha_{1} \cap \alpha_{2} \in K_{p}$ and consequently, $\beta \cap[p] \in K$.

Theorem 3.9 Let $(B, K)$ be a constant filter convergence spaces with $p \in B$. The following are equivalent:

(1) $(B, K)$ is $S T_{3}{ }^{\prime}$ at $p$,

(2) $(B, K)$ is $T_{3}{ }^{\prime}$ at $p$,

(3) $[x] \cap[p] \notin K$ for $x \in B, p \in F$ with $x \neq p$ and $K_{p}=\{[p]\}$, where $\emptyset \neq F \subset B$ is closed with $p \notin F$ and $K_{p}=\{\alpha: \alpha \subset[p]$ and $\alpha \in K\}$.

Proof. By Lemma 3.7 and by Definition 3.3, $(B, K)$ is $S T_{3}{ }^{\prime}$ at $p$ iff $(B, K)$ is $T_{3}{ }^{\prime}$ at $p$. Hence $(1) \Leftrightarrow(2)$.

Suppose $(B, K)$ is $T_{3}{ }^{\prime}$ at $p$. By Definition 3.3, in particular, $(B, K)$ is $T_{1}$ at $p$ and by Theorem 3.6(1), $[x] \cap$ $[p] \notin K, \forall x \in B$ with $x \neq p$.

Suppose $\alpha \in K$ with $\alpha \subset[p]$ and $\emptyset \neq F \subset B$ is closed with $p \notin F$, then it follows that $q \alpha \in K^{\prime}$ and $q \alpha \subset$ $[q(p)]=[p]$. Since $(B, K)$ is $T_{3}{ }^{\prime}$ at $p,\left(B / F, K^{\prime}\right)$ is $p r e T_{2}{ }^{\prime}$ at $p$ for $\emptyset \neq F \subset B$ closed with $p \notin F$, by Theorem 3.6(3), $q \alpha=[p]$ and consequently, by Remark 3.4(3),

$$
\alpha \supset q^{-1} q \alpha=\left[q^{-1}(p)\right]=[p] .
$$

Hence, $\alpha=[p]$, i.e., $K_{p}=\{[p]\}$. 
Suppose (3) holds. We show that $(B, K)$ is $T_{3}{ }^{\prime}$ at $p$. Suppose $x \in B$ with $x \neq p$. If $p \in F$, then by assumption, $[x] \cap[p] \notin K$. If $p \notin F$ and $[x] \cap[p] \in K$, then $[x] \cap[p] \in K_{p}$ and by assumption, $[x] \cap[p]=[p]$ which means $x=p$, a contradiction. Thus, $[x] \cap[p] \notin K, \forall x \in B$ with $x \neq p$. By Theorem 3.6(1), $(B, K)$ is $T_{1}$ at $p$.

Next, we show that $\left(B / F, K^{\prime}\right)$ is $\operatorname{pre}_{2}{ }^{\prime}$ at $p$ for $\emptyset \neq F \subset B$ closed with $p \notin F$. Suppose $\alpha \in K_{p}{ }^{\prime}$. By Definition 2.2, there exists $\beta \in K$ such that

$$
q \beta \subset \alpha \subset[p]
$$

and by Lemma 3.5(1), $\beta \subset[p]$ (since $p \notin F)$. Hence, $\beta \in K_{p}$ and by assumption, $\beta=[p]$. It follows that $q(\beta)=[p] \subset \alpha$ and consequently, $\alpha=[p]$. Hence, $K_{p}{ }^{\prime}=\{[p]\}$ and by Theorem 3.6(3), $\left(B / F, K^{\prime}\right)$ is $\operatorname{preT}_{2}{ }^{\prime}$ at $p$. Hence, by Definition 3.3, $(B, K)$ is $T_{3}{ }^{\prime}$ at $p$.

Let $\mathcal{E}$ be a topological category, $X$ is an object of $\mathcal{E}$ with $p \in U(X)$. Note that by [3,12] if $X$ is $\bar{T}_{0}$ at $p$ and $\operatorname{preT}_{2}{ }^{\prime}$ (resp. pre $\bar{T}_{2}$ ) at $p$, then $X$ is called $L T_{2}$ (resp. $\bar{T}_{2}$ ) at $p$.

Remark 3.10 (1) Let $\boldsymbol{T o p}$ be the category of topological spaces and $(B, \tau) \in \boldsymbol{T o p}$ with $p \in B$.

(i) By Remark 3.2, $L T_{2}$ at $p$ and $\bar{T}_{2}$ at $p$ are same and reduces to $T_{2}$ at $p$, i.e., every $x \in B, x \neq p$, then the points $x$ and $p$ have disjoint neighborhoods [8],

(ii) $T_{3}{ }^{\prime}$ at $p \Leftrightarrow S T_{3}{ }^{\prime}$ at $p \Leftrightarrow \bar{T}_{3}$ at $p \Leftrightarrow S \bar{T}_{3}$ at $p \Rightarrow L T_{2}$ at $p \Leftrightarrow \bar{T}_{2}$ at $p \Rightarrow T_{1}$ at $p \Rightarrow \bar{T}_{0}$ at $p$,

(iii) Let $\boldsymbol{T}_{\mathbf{3}}$ Top be the full subcategory of Top consisting of all local $T_{3}$ topological spaces. By Theorem

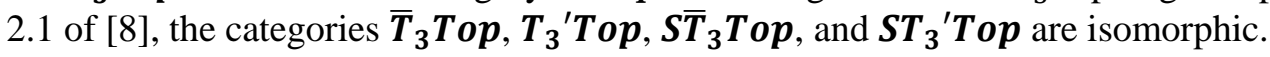

(2) Let $(B, K) \in$ ConFCO with $p \in B$.

(i) By Theorems 3.8 and 3.9,

$$
T_{3}{ }^{\prime} \text { at } p \Leftrightarrow S T_{3}{ }^{\prime} \text { at } p \Rightarrow \bar{T}_{3} \text { at } p \Leftrightarrow \overline{S T}_{3} \text { at } p,
$$

(ii) By Theorems 3.6 and 3.8,

$$
S T_{3}{ }^{\prime} \text { at } p \Rightarrow S \bar{T}_{3} \text { at } p \Rightarrow \bar{T}_{2} \text { at } p \Rightarrow T_{1} \text { at } p \Leftrightarrow \bar{T}_{0} \text { at } p,
$$

(iii) By (ii) and Theorems 3.6 and 3.9,

$$
T_{3}{ }^{\prime} \text { at } p \Rightarrow L T_{2} \text { at } p \Leftrightarrow \operatorname{preT}_{2}{ }^{\prime} \text { at } p \Rightarrow \bar{T}_{2} \text { at } p \Rightarrow p r e \bar{T}_{2} \text { at } p
$$

but converse of each implication is not true. Take $R$, the set of reel numbers and $K=F(R)$. By Theorem 3.6, $(R, F(R))$ is $\operatorname{pre} \bar{T}_{2}$ at $p$ for each $p \in R$ but it is not $\bar{T}_{2}$ at $p$.

Let $B=\{x, y, z\}$ and $K=\{[x],[y],[z],[\varnothing],[x] \cap[y]\}$.

By Theorem 3.4 of [12] and Theorem 3.6, $(B, K)$ is $\bar{T}_{2}$ at $z$ but $(B, K)$ is not $\operatorname{preT}_{2}{ }^{\prime}$ at $z$.

(iv) Let $\boldsymbol{T}_{3}$ ConFCO be the full subcategory of ConFCO whose objects are local $T_{3}$ constant filter convergence spaces, where $T_{3}=T_{3}{ }^{\prime}, \bar{T}_{3}, S \bar{T}_{3}$, and $S T_{3}^{\prime}$. By Theorems 3.8 and 3.9,

(a) $\bar{T}_{3}$ ConFCO and $S \bar{T}_{3}$ ConFCO are isomorphic categories,

(b) $\mathrm{T}_{3}{ }^{\prime} \mathrm{ConFCO}$ and $\mathrm{ST}_{3}{ }^{\prime} \mathrm{ConFCO}$ are isomorphic categories, 
(3) Let $\mathcal{E}$ be a normalized topological category and $X$ be an object of $\mathcal{E}$ with $p \in U(X)$.

(i) By Theorem 7 of [12], if $X$ is $L T_{2}$ at $p$, then $X$ is $\bar{T}_{2}$ at $p$ and by Theorems 2.7 and 2.8 of [10], if $X$ is pre $T_{2}^{\prime}$ at $p$, then $X$ is $p r e \bar{T}_{2}$ at $p$. Moreover, by Theorem 2.8 of [10], if $X$ is $\bar{T}_{3}$ (resp. $S \bar{T}_{3}, T_{3}^{\prime}, S T_{3}{ }^{\prime}$ ), then $X$ is $\bar{T}_{3}$ at $p$ (resp. $S \bar{T}_{3}$ at $p, T_{3}^{\prime}$ at $p, S T_{3}^{\prime}$ at $p$ ).

(ii) Note that all objects of a set-based arbitrary topological category may be $p r e \bar{T}_{2}$ at $p$. For example, it is shown, in [13], that all Cauchy spaces [14] are $\operatorname{pre} \bar{T}_{2}$ at $p$. Also, $p r e T_{2}^{\prime}$ at $p$ objects could be only discrete objects [15].

(iii) Let $\boldsymbol{p r} \boldsymbol{e} \overline{\boldsymbol{T}}_{2}(\mathcal{E})$ be the full subcategory of $\mathcal{E}$ consisting of all $p r e \bar{T}_{2}$ objects. By Theorem 3.4 of [16], $\operatorname{preT}_{2}(\mathcal{E})$ is a topological category.

Theorem 3.11 (1) If a constant filter convergence space $(B, K)$ is $\bar{T}_{3}$ (resp. $\left.T_{3}^{\prime}\right)$ at $p$ and $M \subset B$ with $p \in M$, then $M$ is $\bar{T}_{3}\left(\operatorname{resp} . T_{3}^{\prime}\right)$ at $p$,

(2) For all $i \in I$ and $p_{i} \in B_{i},\left(B_{i}, K_{i}\right)$ is $\bar{T}_{3}$ at $p_{i}$ if $\left(B=\prod_{i \in I} B_{i}, K\right)$ is $\bar{T}_{3}$ at $p=\left(p_{1}, p_{2}, \ldots\right)$, where $K$ is the product structure on $B$.

Proof. (1) Let $i: M \subset B$ be the inclusion map, $K_{M}$ be a structure on $M$ induced from $i$, and $[x] \cap[p] \in K_{M}$ for $x \in M$ with $x \neq p$. By Definition 2.1,

$$
i([x] \cap[p])=i([x]) \cap i([p])=[x] \cap[p] \in K
$$

for $x \in X$ with $x \neq p$, a contradiction since $(B, K)$ is $\bar{T}_{3}$ (resp. $T_{3}^{\prime}$ ) at $p$. Thus, $[x] \cap[p] \notin K_{M}$ for $x \in M$. with $x \neq p$.

Suppose $\alpha, \beta \in\left(K_{M}\right)_{p}$. Then $i(\alpha), i(\beta) \in K, i(\alpha) \subset[p], i(\beta) \subset[p]$ and by Theorem 3.8, $i(\alpha \cap \beta) \in K_{p}$. By Definition 2.1, $\alpha \cap \beta \in\left(K_{M}\right)_{p}$.

Suppose $\alpha \in\left(K_{M}\right)_{p}, \beta \in K_{M}$ and for $\emptyset \neq F \subset M$ closed with $p \notin F$ such that $\alpha \cup \beta$ is proper or $\beta \cup[F]$ and $\alpha \cup[F]$ are proper.

By Definition 2.1,i( $\alpha), i(\beta) \in K_{p}, i(\alpha) \cup i(\beta)=i(\alpha \cup \beta)$ is proper or

$$
i(\alpha) \cup[i(F)=F]
$$

and

$$
i(\beta) \cup[i(F)=F]
$$

are proper. By Theorem 3.8, $i(\beta \cap[p]) \in K$ and by Definition 2.1, $\beta \cap[p] \in K_{M}$. Hence, $\left(M, K_{M}\right)$ is $\bar{T}_{3}$ at $p$. It remains to show that $\left(K_{M}\right)_{p}=\{[p]\}$.

Let $\alpha \in\left(K_{M}\right)_{p}$ and for $\emptyset \neq F \subset M$ closed with $p \notin F$. By Definition 2.1, $i(\alpha) \in K_{p}$ and by Theorem 3.9, $K_{p}=\{[p]\}$.

Thus, $i(\alpha)=[p]$ and Definition 2.1, $\alpha=[p]$. Hence, $\left(M, K_{M}\right)$ is $T_{3}^{\prime}$ at $p$.

(2) Suppose that $\left(B=\prod_{i \in I} B_{i}, K\right)$ is $\bar{T}_{3}$ at $p$. Since each $\left(B_{i}, K_{i}\right)$ is isomorphic to a subspace of $(B, K)$, by Part (1), $\forall i \in I,\left(B_{i}, K_{i}\right)$ is $\bar{T}_{3}$ at $p_{i}$. 


\section{ACKNOWLEDGEMENTS}

This paper was presented in the 14th Ankara Mathematics Days Symposium held on June 28-29, 2019.

\section{CONFLICTS OF INTEREST}

No conflict of interest was declared by the authors.

\section{REFERENCES}

[1] Cartan, H., “Theorie des filtres”, C. R. de l'Academie des Sciences, 205:595-598, (1937).

[2] Cartan, H., "Filtres et ultrafiltres", C. R. de l'Academie des Sciences, 205:777-779, (1937).

[3] Schwarz, F.,"Connections between convergence and nearness", The Series Lecture Notes in Mathematics, 719:345-354, (1978).

[4] Baran, M., "Separation properties", Indian J. Pure Applied Mathematics, 23:333-341, (1991).

[5] Baran, M., "The notion of closedness in topological categories", Commentationes Mathematicae Universitatis Carolinae, 34:383-395, (1993).

[6] Adamek, J., Herrlich, H., and Strecker, G. E., Abstract and concrete categories, John Wiley and Sons, New York, (1990).

[7] Nel, L. D., "Initially structured categories and cartesian closedness", Canadian Journal of Mathematics, 27:1361-1377, (1975).

[8] Baran, M., "Separation properties in topological categories", Mathematica Balkanica, 10:39-48, (1996).

[9] Baran, M., "Stacks and filters", Turkish Journal of Mathematics, 16:95-108, (1992).

[10] Baran, M., " $T_{3}$ and $T_{4}$ objects in topological categories", Indian Journal Pure Applied Mathematics, 29:59-69, (1998).

[11] Erciyes, A., \& Baran, M., "Local pre-Hausdorff constant filter convergence spaces", Turkish Journal of Mathematics and Computer Science, 10:82-87, (2018).

[12] Baran, T. M., "Local $T_{2}$ extended pseudo-quasi-semi metric spaces", Communications Faculty of Sciences University of Ankara Series A1 Mathematics and Statistics, 68(2):2117-2127, (2019).

[13] Kula, M., "Separation properties at $p$ for the topological category of cauchy spaces", Acta Mathematica Hungarica, 136:1-15, (2012).

[14] Lowen-Colebunders, E., "Function classes of cauchy continuous maps", New York USA, Marcel Dekker Inc., (1989).

[15] Baran, T. M., \& Kula, M., "Local pre-Hausdorff extended pseudo-quasi-semi metric spaces", Communications Faculty of Sciences University of Ankara Series A1 Mathematics and Statistics, 68(1):862-870, (2019).

[16] Baran, M., "Pre $T_{2}$ Objects in topological categories", Applied Categorical Structures, 17:591$602,(2009)$. 